\title{
Embeddings of diffeomorphisms of the plane in regular iteration semigroups
}

\author{
Marek Cezary Zdun and PaWę Solarz \\ Dedicated to Professor János Aczél on his 90th birthday
}

\begin{abstract}
We give the full description of the $C_{\delta}^{r}$ embeddings of a given diffeomorphism $F: \mathbb{R}^{2} \supset U \rightarrow \mathbb{R}^{2}$ of class $C^{r}$ such that $F(0)=0$ and $d^{(r)} F(x)=d^{(r)} F(0)+O\left(\|x\|^{\delta}\right),\|x\| \rightarrow$ 0 with a hyperbolic fixed point. That is we determine all families of $C_{\delta}^{r}$ diffeomorphisms of the plane defined in a neighbourhood of the origin such that $F^{t} \circ F^{s}=F^{t+s}, t, s \geq 0, F^{1}=F$ and the mapping $t \mapsto F^{t}(x)$ is continuous. To describe these semigroups we determine the real logarithms and all continuous groups of the real non-singular matrices.
\end{abstract}

Mathematics Subject Classification (2010). Primary 39B12; Secondary 26A18 · 37C15.

Keywords. Iteration semigroup $\cdot$ Semiflows $\cdot C_{\delta}^{r}$-embedding $\cdot$ Real logarithm of matrix . Semigroup of matrices · Functional equation.

\section{Introduction}

In [16] the author considered the problem of the embeddability of a given diffeomorphism $F: U \rightarrow U$ in $C^{r}$-iteration semigroups, where $0 \in U \subset \mathbb{R}^{N}$, $N \geq 2$ and 0 is the only hyperbolic fixed point of $F$. The main result gives the conditions for the embeddability of $F$ in a regular iteration semigroup. In this note we study the particular case $N=2$ which has its own specificity being a consequence of some geometrical and algebraical properties of the plane. The general assumptions made in [16] here are simply verifiable and we obtain a readable form for the given formulas. Moreover, we generalize the main result from [16] replacing the class of functions $C^{r}$ by a more general class $C_{\delta}^{r}$ and dispose of the assumption of the lake of resonances (see [1]). Also in the planar case we are able to give a clear description of the trajectory of the considered flows. The astonishing property that linear mappings $F(x)=\lambda x$ have in the plane which possess very rich families of $C_{\delta}^{1}$ embeddings which is not true in dimension 1 (see Theorem 2.4 case (iii)). 
Let $r \geq 1$ be an integer, $0 \leq \delta \leq 1$ and let $U \subset \mathbb{R}^{2}$ be a neighbourhood of 0 . By $\operatorname{Diff}_{\delta}^{r}(U)$ denote the set of all diffeomorphisms $F: U \rightarrow \mathbb{R}^{2}$ of class $C^{r}$ such that $F(0)=0$ and

$$
d^{(r)} F(x)=d^{(r)} F(0)+O\left(\|x\|^{\delta}\right), \quad\|x\| \rightarrow 0 .
$$

We call $F$ a $C_{\delta}^{r}$ mapping if $F \in \operatorname{Diff}_{\delta}^{r}(U)$. A family of $C_{\delta}^{r}$ mappings $F^{t}: U \rightarrow U$, $t \geq 0$ is said to be a $C_{\delta}^{r}$ iteration semigroup or a $C_{\delta}^{r}$ semiflow if:

$$
F^{t} \circ F^{s}=F^{t+s}, \quad t, s \geq 0
$$

and for every $x \in U$ the mapping $t \mapsto F^{t}(x)$ is Lebesgue measurable. If $F^{1}=F$ then this semigroup is said to be a $C_{\delta}^{r}$ embedding of $F$ or a $C_{\delta}^{r}$ iteration semigroup of $F$. We also say that $F$ is $C_{\delta}^{r}$ embeddable.

In this paper we determine all continuous groups of a real non-singular matrix $S \in M_{2}(\mathbb{R})$. We then apply these results and generalize the results from [16] to give the full description of the regular iteration semigroups of the given diffeomorphism defined on some neighbourhood of the hyperbolic fixed point 0 . In order to do this we determine all real logarithms of a real non-singular matrix $S \in M_{2}(\mathbb{R})$.

We say that a real square matrix $S$ has a real logarithm if there exists a real matrix $A$ such that

$$
S=e^{A}
$$

where $e^{A}=\sum_{n=0}^{\infty} \frac{1}{n !} A^{n}$.

It is known that every non-singular real matrix $S$ has a complex logarithm (see [15]), however, $S$ has a real logarithm if and only if $S$ is non-singular and the geometric multiplicity of each real negative eigenvalue $\lambda$ of $S$ is even (see [4], [12]). This is equivalent to the property that each Jordan block of $S$ belonging to a negative eigenvalue repeats an even number of times. Moreover, such a logarithm is unique if and only if all eigenvalues of $S$ are real and positive and their geometric multiplicities are equal to one, i.e. no Jordan blocks of $S$ belonging to the same eigenvalues appear more than once (see $[4,12])$.

Let $I$ stand for the identity matrix. By $\sigma(S)$ denote the set of eigenvalues of $S$. The above facts in the particular case may be put as follows

Proposition 1.1. A matrix $S \in M_{2}(\mathbb{R})$ has a real logarithm if and only if $S$ is non-singular and $\sigma(S) \subset \mathbb{C} \backslash \mathbb{R}^{-}$or $S=\lambda I$ for some $\lambda<0$. This logarithm is unique if and only if $\sigma(S) \subset \mathbb{R}^{+}$and $S \neq \lambda I$ for some $\lambda \in \mathbb{R}^{+}$.

In Appendix 3 we determine explicitly all real logarithms of the above matrices. These logarithms are applied in the description of matrix embeddings.

Recall that a family $\mathcal{S}=\left\{S^{t} \in M_{n}(\mathbb{R}), t \in \mathbb{R}\right\}$ is called a continuous iteration group if $S^{t} S^{s}=S^{t+s}$ for $t, s \in \mathbb{R}$ and $\lim _{t \rightarrow 0} S^{t}=I$. If $S^{1}=S$ for a given matrix $S$ we call $\mathcal{S}$ a linear embedding of $S$. 
Suppose that $S \in M_{2}(\mathbb{R})$ has a real logarithm $A$, i.e. $S=e^{A}$ and put

$$
S^{t}:=e^{t A}, \quad t \in \mathbb{R} .
$$

Then the family $\left\{S^{t}, t \in \mathbb{R}\right\}$ is a continuous embedding of $S$. Conversely, if $\left\{S^{t}, t \in \mathbb{R}\right\}$ is a continuous iteration group of linear mappings then there exists a limit $\lim _{t \rightarrow \infty} \frac{1}{t}\left(S^{t}-I\right)=: A$ and (1.2) holds (see [5] p.283). Hence $A \in M_{2}(\mathbb{R})$. Thus every group $\left\{S^{t}, t \in \mathbb{R}\right\}$ determines uniquely the real matrix $A$ such that $e^{A}=S^{1}$.

By $P=[\mathbf{a}, \mathbf{b}] \in M_{2}(\mathbb{R})$ we denote a matrix with two linearly independent columns $\mathbf{a}, \mathbf{b} \in \mathbb{R}^{2}$. We also set

$$
W(t):=\left[\begin{array}{cc}
\cos t & \sin t \\
-\sin t & \cos t
\end{array}\right] .
$$

The basis of our considerations is the following.

Proposition 1.2. Let $S \in M_{2}(\mathbb{R})$ be a non-singular matrix.

(i) If $S$ has two distinct real eigenvalues $\lambda_{1}, \lambda_{2}>0$ then $S$ has a unique real linear embedding and

$$
S^{t}=P\left[\begin{array}{cc}
\lambda_{1}^{t} & 0 \\
0 & \lambda_{2}^{t}
\end{array}\right] P^{-1},
$$

where $S \mathbf{a}=\lambda_{1} \mathbf{a}$ and $S \mathbf{b}=\lambda_{2} \mathbf{b}$.

(ii) If $S$ has a unique eigenvalue $\lambda>0$ and $S \neq \lambda I$ then $S$ has a unique real linear embedding. This embedding is given by the formula

$$
S^{t}=\lambda^{t} P\left[\begin{array}{ll}
1 & \frac{t}{\lambda} \\
0 & 1
\end{array}\right] P^{-1},
$$

where $S \mathbf{a}=\lambda \mathbf{a}$ and $S \mathbf{b}=\lambda \mathbf{a}+\mathbf{b}$.

(iii) If $S=\lambda I$ then $S$ has infinitely many real linear embeddings. They are given by the formula

$$
S^{t}=|\lambda|^{t} R W(t k \pi) R^{-1}, \quad k \in \mathbb{Z},
$$

where $R$ is an arbitrary non-singular matrix, $k$ is odd if $\lambda<0$ and $k$ is even if $\lambda>0$.

(iv) If $S$ has an eigenvalue $\lambda \in \mathbb{C} \backslash \mathbb{R}$ then $S$ has countably many real linear embeddings. These embeddings are given by

$$
S^{t}=|\lambda|^{t} P W(t(\operatorname{Arg} \lambda+2 k \pi)) P^{-1}, \quad k \in \mathbb{Z},
$$

where $\mathbf{a}=\operatorname{Re} \mathbf{w}, \mathbf{b}=\operatorname{Im} \mathbf{w}$ and $S \mathbf{w}=\lambda \mathbf{w}$.

If $S$ is linearly embeddable then $S$ satisfies one of the assumptions from the cases (i), (ii), (iii) or (iv) above.

The proof is a direct consequence of Proposition 3.1 from Appendix 3.

Let us note that another approach to the determination of all iteration groups of real square matrices is given also in [6] and [2]. In [6] the problem 
is solved in the most general case, i.e. without any regularity with respect to the parameter $t$ and the solution is obtained by the methods of functional equations.

\section{The embeddings of diffeomorphisms}

In this section we assume that

(H) $F \in \operatorname{Diff}_{\delta}^{r}(U), r+\delta>1$ and $\lambda_{1}, \lambda_{2}$ are eigenvalues of $S:=d F(0)$ satisfying $\left|\lambda_{1}\right| \leq\left|\lambda_{2}\right|<1$.

The problem of embedding a mapping $F \in \operatorname{Diff}_{\delta}^{r}(U)$ in a regular iteration semigroup is strictly connected to the problem of the existence of the real embedding of the matrix $S=d F(0)$ (see Proposition 1.2) and the problem of the existence of the formal solution of

$$
\varphi(F(x))=S \varphi(x), \quad x \in U .
$$

Recall that a polynomial function $\eta_{r}: \mathbb{R}^{2} \rightarrow \mathbb{R}^{2}(r \geq 2)$ given by $\eta_{r}(x)=$ $x+\sum_{k=2}^{r} L_{k}(x)$, where $L_{k}$ are homogeneous polynomials of degree $k$, is said to be a formal solution of (2.1) of $r$-th order if it satisfies the system

$$
d^{(k)}\left(\eta_{r} \circ F-S \eta_{r}\right)(0)=0, \quad k=2,3, \ldots, r .
$$

Such a solution exists and is unique if $F$ has no resonance up to $r$-order (see e.g. $[13,14]$ ), which in the two dimensional case means that the eigenvalues of $S$ satisfy $\lambda_{2}^{k} \neq \lambda_{1}$ for an integer $2 \leq k \leq r$ (see [17]).

Theorem 2.1. Let $F$ satisfy $(\mathrm{H}),\left|\lambda_{2}\right|^{r+\delta}<\left|\lambda_{1}\right|$ and $\lambda_{2}^{k} \neq \lambda_{1}$ for $2 \leq k \leq r$ (if $r \geq 2)$. Then $F$ has a $C_{\delta}^{r}$ embedding $\left\{F^{t}: V \rightarrow V, t \geq 0\right\}$, where $V \subset U$ is a neighbourhood of 0 if and only if $S$ has a real logarithm.

This embedding is of the form

$$
F^{t}(x)=\varphi^{-1}\left(S^{t} \varphi(x)\right), \quad t \geq 0,
$$

where $\left\{S^{t}, t \in \mathbb{R}\right\}$ is a continuous linear embedding of $S$ and $\varphi$ is given by the formula

$$
\varphi(x)=\lim _{n \rightarrow \infty} S^{-n} \circ \theta_{r} \circ F^{n}(x),
$$

where $\theta_{r}=i d$ for $r=1, \delta>0$ and $r=2$ with $\delta=0, \theta_{r}=\eta_{r-1}$ for $r \geq 2$ and $\delta=0$ and $\theta_{r}=\eta_{r}$, otherwise, here $\eta_{r}$ is a formal solution of $r$-th order of (2.1).

Proof. If $r \geq 2$ then (2.1) has a formal solutions $\eta_{k}$ up to $r$-order. It follows by Kuczma's theorem on linearization (see [8] and [9] p. 336) that Eq. (2.1) has a unique solution $\varphi \in \operatorname{Diff}_{\delta}^{r}(W)$, where $W \subset U$ is a basin of attraction of 0 such that $d \varphi(0)=I$ and $d^{(k)} \varphi(0)=\eta_{k}$ for $k=2, \ldots, r$ (if $r \geq 2$ ). 
In the cases $r=1$ with $\delta>0$ and $r=2$ with $\delta=0$ it follows respectively by Theorems 1 and 2 in [17] that $\varphi$ is given by the formula

$$
\varphi(x)=\lim _{n \rightarrow \infty} S^{-n} \circ F^{n}(x) .
$$

If $r \geq 3$ and $\delta=0$ then by Theorem 3 in [17],

$$
\varphi(x)=\lim _{n \rightarrow \infty} S^{-n} \circ \eta_{r-1} \circ F^{n}(x),
$$

however if $r \geq 2$ and $\delta>0$ then Theorem 1 in [17] implies that

$$
\varphi(x)=\lim _{n \rightarrow \infty} S^{-n} \circ \eta_{r} \circ F^{n}(x) .
$$

Let $S$ have a real logarithm $A$. Put $S^{t}:=e^{t A}, t \geq 0$. Similarly as in the proof of Theorem 1 in [16] one can show that there exists an open set $B \subset \varphi[W]$ such that $0 \in S^{t}[B] \subset B$ for $t \geq 0$. This property enables us to define the family of functions $(2.2)$ on the set $V:=\varphi^{-1}[B]$. Obviously (2.2) defines a $C_{\delta}^{r}$ iteration semigroup on $V$ such that $F^{1}=F$ and $d F^{t}(0)=S^{t}$ for $t \geq 0$.

Now we prove the uniqueness. Let $\left\{G^{t}: V \rightarrow V, t \geq 0\right\}$ be a $C_{\delta}^{r}$ iteration semigroup of $F$. Define $S^{t}:=d F^{t}(0)$. Obviously $\left\{S^{t}, t \geq 0\right\}$ is a continuous linear embedding of $S$. Hence there exists a $\operatorname{limit}_{t \rightarrow \infty} \frac{1}{t}\left(S^{t}-I\right)=: A$ and $A$ is a real logarithm of $S$ (see [5] p. 283).

Put $\Phi_{t}:=\left(S^{t}\right)^{-1} \circ \varphi \circ G^{t}, t \geq 0$. Obviously $\Phi_{t}$ are of class $C^{r}$ and $d \Phi_{t}(0)=I$. By (2.1) and the fact that $G^{1}=F$ we have

$$
\begin{aligned}
\Phi_{t} \circ F & =\left(S^{t}\right)^{-1} \circ \varphi \circ G^{t} \circ F=\left(S^{t}\right)^{-1} \circ \varphi \circ G^{t} \circ G^{1} \\
& =\left(S^{t}\right)^{-1} \circ \varphi \circ G^{1} \circ G^{t}=\left(S^{t}\right)^{-1} \circ \varphi \circ F \circ G^{t} \\
& =\left(S^{t}\right)^{-1} \circ S \circ \varphi \circ G^{t}=S \circ\left(S^{t}\right)^{-1} \circ \varphi \circ G^{t}=S \Phi_{t}, \quad t \geq 0 .
\end{aligned}
$$

By Theorem 1 in [17] $\varphi$ is the unique $C_{\delta}^{r}$ solution of (2.1) such that $d \varphi(0)=I$. Hence $\varphi(x)=\Phi_{t}(x)=\left(S^{t}\right)^{-1} \circ \varphi \circ G^{t}(x)$ for $x \in V$ and $t \geq 0$. Consequently, $S^{t} \varphi(x)=\varphi\left(G^{t}(x)\right)$ so $G^{t}(x)=\varphi^{-1}\left(S^{t} \varphi(x)\right)$. This and (2.2) yield $F^{t}(x)=$ $G^{t}(x), t \geq 0, x \in V$.

By the same arguments we also get the more general statement.

Remark 2.2. If $U \subset \mathbb{R}^{N}$ and $\lambda_{1}, \ldots, \lambda_{N}$ are the eigenvalues of $d F(0)$ such that $\left|\lambda_{1}\right| \leq \ldots,\left|\lambda_{N}\right| \leq 1$ and $\left|\lambda_{N}\right|^{r+\delta}<\left|\lambda_{1}\right|$, then the same assertion as in Theorem 2.1 holds.

This result generalizes Theorem 1 in [16]. However, in the discrete case, i.e. for $t \in \mathbb{N}$ this generalizes the Sternberg theorem (see [14]). Note that it follows from the Hartman-Grobman theorem (see [11]) that the formula (2.2) with a homeomorphism $\varphi$ occurs for $C^{1}$ iteration semigroups but the function $\varphi$ although satisfies Eq. (2.1) is not uniquely determined.

Let us note the following obvious statement. 
Remark 2.3. Let $\varphi: U \rightarrow \mathbb{R}^{2}$ be a diffeomorphism such that $\varphi(0)=0, d \varphi(0)=$ $I$. Suppose that $S^{t}, P$ and $J^{t}$ are non-singular matrices such that $S^{t} P=$ $P J^{t}$ and let $F: U \rightarrow U$ be a mapping. Put $\psi:=P^{-1} \varphi$, then (i) $F^{t}(x)=$ $\varphi^{-1}\left(S^{t} \varphi(x)\right)$ if and only if $F^{t}(x)=\psi^{-1}\left(J^{t} \psi(x)\right)$ for $x \in U$ and $t \geq 0$ and (ii) $\varphi(x)=\lim _{n \rightarrow \infty} S^{-n} F^{n}(x)$ if and only if $\psi(x)=\lim _{n \rightarrow \infty} P^{-1} S^{-n} F^{n}(x)$ $=\lim _{n \rightarrow \infty} J^{-n} P F^{n}(x)$ for $x \in U$.

Let a matrix $P$ be such that $P^{-1} S P$ is a real Jordan form of $S$. By Theorem 2.1, Remark 2.3 and Proposition 1.2 we get the following results

Theorem 2.4. Suppose that $F$ satisfies $(\mathrm{H})$.

(i) If $\lambda_{1} \neq \lambda_{2}, \lambda_{2}^{r+\delta}<\lambda_{1}<\lambda_{2}^{r-1}$ and $\lambda_{2}^{r} \neq \lambda_{1}$ then the mapping $F$ is $C_{\delta}^{r}$ embeddable if and only if $\lambda_{1}, \lambda_{2}>0$. Moreover, $F$ has a unique $C_{\delta}^{r}$ embedding $\left\{F^{t}: V \rightarrow V, t \geq 0\right\}$ in a neighbourhood of the origin. It is given by the formula

$$
F^{t}(x)=\psi^{-1}\left(\lambda_{1}^{t} \psi_{1}(x), \lambda_{2}^{t} \psi_{2}(x)\right), \quad x \in V, t \geq 0,
$$

where $\psi=\left(\psi_{1}, \psi_{2}\right)=P^{-1} \varphi$ and $\varphi$ is given by (2.3).

(ii) If $\lambda \in \mathbb{R} \backslash\{0\}$ is a unique eigenvalue of $S$ and $S \neq \lambda I$ then the function $F$ is $C_{\delta}^{r}$ embeddable if and only if $\lambda>0$. Then $F$ has exactly one $C_{\delta}^{r}$ embedding. This embedding is of the form

$F^{t}(x)=\psi^{-1}\left(\lambda^{t}\left(\psi_{1}(x)+\frac{t}{\lambda} \psi_{2}(x)\right), \lambda^{t} \psi_{2}(x)\right), \quad x \in V, t \geq 0$,

where $V \subset U$ is a neighbourhood of the origin, $\psi=\left(\psi_{1}, \psi_{2}\right)=P^{-1} \varphi$ and $\varphi$ is given by (2.3).

(iii) If $d F(0)=\lambda I$, where $\lambda \in \mathbb{R} \backslash\{0\}$ then $F$ has infinitely many $C_{\delta}^{r}$ embeddings $\left\{F^{t}: V \rightarrow V, t \geq 0\right\}$, where $V \subset U$ is a neighbourhood of the origin and

$$
F^{t}(x)=\psi^{-1}\left(|\lambda|^{t} W(t k \pi) \psi(x)\right), \quad x \in V, t \geq 0,
$$

where $\psi=R \varphi, \varphi$ is given by (2.3) and $R$ is an arbitrary non-singular matrix, $k \in \mathbb{Z}$ and $k$ is odd if $\lambda<0$ and $k$ is even if $\lambda>0$.

(iv) If $\lambda \in \mathbb{C} \backslash \mathbb{R}$ is the eigenvalue of $S$ then $F$ has countably many $C_{\delta}^{r}$ embeddings. These embeddings are given by the formula

$$
F^{t}(x)=\psi^{-1}\left(|\lambda|^{t} W(t(\operatorname{Arg} \lambda+2 k \pi)) \psi(x)\right), \quad x \in V, t \geq 0
$$

for $k \in \mathbb{Z}$, and some neighbourhood $V \subset U$ of the origin, where $\psi=$ $P^{-1} \varphi$ and $\varphi$ is given by (2.3).

Proof. (i) Let us note that $F$ has no resonance up to $r$-th order. Let $P$ be a matrix such that

$$
S=P\left[\begin{array}{cc}
\lambda_{1} & 0 \\
0 & \lambda_{2}
\end{array}\right] P^{-1} .
$$


Put $\psi=P^{-1} \varphi$, where $\varphi$ is given by (2.3). By Theorem 2.1 and Remark 2.3 we get our assertion.

(ii) Here $\lambda_{1}=\lambda_{2}=\lambda$, so $\lambda_{2}^{r+\delta}<\lambda_{1}$ as $r+\delta>1$. Moreover, $F$ has no resonance of any order. Thus, by Theorem 2.1, Proposition 1.2 (ii) and Remark 2.3, we get our assertion. The formula for $\psi$ follows from Theorem 2.1 for $r=1$ and $\delta>0$, since $\lambda_{2}^{1+\delta}<\lambda_{1}$.

(iii) The argumentation is the same as in (ii) above but here we apply Proposition 1.2 (iii).

Similarly, in view of Proposition 1.2 case (iv), we obtain (iv).

Remark 2.5. Theorem 2.4 is true also in the $N$ dimensional case under the additional condition that $F$ has no resonance up to $r$-th order and $d F(0)$ has exactly $N$ eigenvalues $0<\lambda_{1}<\ldots<\lambda_{N}<1$ and $\lambda_{N}^{r+\delta}<\lambda_{1}<\lambda_{N}^{r}$. In the cases $r=1, \delta>0$ and $r=2, \delta=1$ the assumption on the resonance can be omitted. The proof is the same.

Remark 2.6. If $F$ is of class $C_{\delta}^{1}$ with $\delta>0$ and $S$ has either complex eigenvalues or only one eigenvalue, then the function $\psi$ in Theorem 2.4 (ii), (iii), (iv) is given by $\psi(x)=P^{-1} \lim _{n \rightarrow \infty} S^{-n} \circ F^{n}(x)$. If $\delta=0$ then the $C^{1}$ embedding depends on an arbitrary function (see [7]).

By Theorems 2.1 and 2.4 (iii) we obtain.

Theorem 2.7. Let $U=(-a, a)$ and $f_{i}: U \rightarrow U$ be the injections belonging to the class $\operatorname{Diff}_{\delta}^{r}(U)$ for $r \geq 1$ and $\delta>0, f_{i}(0)=0, f_{i}(x) \neq x$ for $x \neq 0$ and $f_{i}^{\prime}(0)=\lambda_{i}$ for $i=1,2$ and $F(x, y):=\left(f_{1}(x), f_{2}(y)\right), x, y \in U$. If $\lambda_{1} \neq \lambda_{2}$ and $\lambda_{2}^{r+\delta}<\lambda_{1}$ then $F$ has a unique $C_{\delta}^{r}$ embedding.

If $\lambda_{1}=\lambda_{2}$ then $F$ has uncountably many $C_{\delta}^{r}$ embeddings.

Proof. Suppose $\lambda_{1}, \lambda_{2}>0$. It follows by Theorem 1 in [16] that there exist limits $\psi_{i}(x):=\lim _{n \rightarrow \infty} \frac{f_{i}^{n}(x)}{\lambda_{i}^{n}}, i=1,2$ and they are of class $C_{\delta}^{r}$, moreover $\psi_{i}\left(f_{i}(x)\right)=\lambda_{i} \psi_{i}(x)$ for $i=1,2$ in $U$. The families $f_{i}^{t}(x)=\psi_{i}^{-1}\left(\lambda_{i}^{t} \psi_{i}(x)\right)$ are $C_{\delta}^{r}$ embeddings of $f_{i}$ for $i=1,2$. These embeddings are unique (see [7]). Hence it is easy to verify that $F^{t}(x, y)=\left(f_{1}^{t}(x), f_{2}^{t}(y)\right)$ for $t \geq 0, x, y \in U$ is a $C_{\delta}^{r}$ iteration semigroup. This implies that (2.1) has a formal solution of $r$-th order. It follows by Theorem 2.1 that if $\lambda_{1} \neq \lambda_{2}$, then $F$ possesses a unique $C_{\delta}^{r}$ iteration semigroup $\left\{F^{t}, t \geq 0\right\}$.

If $\lambda_{1}=\lambda_{2}=\lambda$ then, by Theorem 2.4 (iii), the diffeomorphism $F$ possesses infinitely many $C_{\delta}^{r}$ embeddings $\left\{F^{t}, t \geq 0\right\}$. They are given by the formula $F^{t}(x, y)=\left(F_{1}^{t}(x, y), F_{2}^{t}(x, y)\right)$ for $t \geq 0,(x, y) \in U^{2}$, where

$$
\begin{aligned}
& F_{1}^{t}(x, y)=\varphi_{1}^{-1}\left(|\lambda|^{t} \varphi_{1}(x) \cos (2 t k \pi)+|\lambda|^{t} \varphi_{2}(y) \sin (2 t k \pi)\right) \\
& F_{2}^{t}(x, y)=\varphi_{2}^{-1}\left(|\lambda|^{t} \varphi_{2}(y) \cos (2 t k \pi)-|\lambda|^{t} \varphi_{1}(x) \sin (2 t k \pi)\right)
\end{aligned}
$$


for $x, y \in U$ and

$$
\begin{array}{ll}
\varphi_{1}(x)=p_{11} \lim _{n \rightarrow \infty} \frac{f_{1}^{n}(x)}{\lambda^{n}}+p_{12} \lim _{n \rightarrow \infty} \frac{f_{2}^{n}(x)}{\lambda^{n}}, \quad x \in U, \\
\varphi_{2}(x)=p_{21} \lim _{n \rightarrow \infty} \frac{f_{1}^{n}(x)}{\lambda^{n}}+p_{22} \lim _{n \rightarrow \infty} \frac{f_{2}^{n}(x)}{\lambda^{n}}, \quad x \in U
\end{array}
$$

and $P=\left[p_{i j}\right]$ is an arbitrary non-singular real matrix.

\section{Appendix}

In this section we determine the form of all real logarithms of $S \in M_{2}(\mathbb{R})$ giving at the same time the direct proof of Proposition 1.1.

Set

$$
J_{z}(x, y):=\left[\begin{array}{ll}
x & z \\
0 & y
\end{array}\right] \text { and } \quad J(x, y):=\left[\begin{array}{cc}
x & y \\
-y & x
\end{array}\right]
$$

for $x, y, z \in \mathbb{R}$.

Let us recall also that for every matrix $A \in M_{2}(\mathbb{R})$ there is a non-singular matrix $P$ conjugating $A$ with its Jordan form, i.e. $P^{-1} A P=J$, where $J \in$ $\left\{J_{0}(x, y), x I, J_{1}(x, x), J(a, b)\right\}, x, y, a, b \in \mathbb{R}, b \neq 0, x \neq y$ and $x, y, a+\mathrm{i} b$ are the eigenvalues of $A$.

Proposition 3.1. Let $S \in M_{2}(\mathbb{R})$.

(i) If $S$ has two distinct real eigenvalues $\lambda_{1}, \lambda_{2}$ then $S$ has a real logarithm if and only if $\lambda_{1}>0$ and $\lambda_{2}>0$. Such a logarithm is unique. It is given by the formula

$$
A=P J_{0}\left(\ln \lambda_{1}, \ln \lambda_{2}\right) P^{-1},
$$

where $P=[\mathbf{a}, \mathbf{b}]$ and $S \mathbf{a}=\lambda_{1} \mathbf{a}, S \mathbf{b}=\lambda_{2} \mathbf{b}$.

(ii) Let $S$ have a unique eigenvalue $\lambda \in \mathbb{R} \backslash\{0\}$ and $S \neq \lambda I$. Then $S$ has a real logarithm if and only if $\lambda>0$. Such a logarithm is unique. It is given by the formula

$$
A=P J_{\frac{1}{\lambda}}(\ln \lambda, \ln \lambda) P^{-1},
$$

where $P=[\mathbf{a}, \mathbf{b}], S \mathbf{a}=\lambda \mathbf{a}$ and $S \mathbf{b}=\lambda \mathbf{a}+\mathbf{b}$.

(iii) If $\lambda \in \mathbb{R} \backslash\{0\}$ then the matrix $\lambda I$ has infinitely many real logarithms. More precisely, if $e^{A}=\lambda I$ and $A \in M_{2}(\mathbb{R})$ then there are an integer $k$ and a non-singular matrix $P \in M_{2}(\mathbb{R})$ such that

$$
A=P J(\ln |\lambda|, k \pi) P^{-1},
$$

where $k$ is an odd number if $\lambda<0$ and $k$ is an even number if $\lambda>0$. For any non-singular matrix $P \in M_{2}(\mathbb{R})$ equality (3.3) defines a real logarithm of $\lambda I$. 
(iv) If $S$ has an eigenvalue $\lambda \in \mathbb{C} \backslash \mathbb{R}$ then $S$ has a countably many real logarithms. They are given by the formula

$$
A=P J(\ln |\lambda|, \operatorname{Arg} \lambda+2 k \pi) P^{-1}, \quad k \in \mathbb{Z},
$$

where $P=[\mathbf{a}, \mathbf{b}], \mathbf{a}=\operatorname{re} \mathbf{w}, \mathbf{b}=\operatorname{im} \mathbf{w}$ and $S \mathbf{w}=\lambda \mathbf{w}$.

Proof. Case (i). Let $P=[\mathbf{a}, \mathbf{b}]$ and $S \mathbf{a}=\lambda_{1} \mathbf{a}, S \mathbf{b}=\lambda_{2} \mathbf{b}$. Assume that there exists $A \in M_{2}(\mathbb{R})$ such that $e^{A}=S$. Putting $B:=P^{-1} A P$ we have

$$
e^{B}=e^{P^{-1} A P}=P^{-1} S P=J_{0}\left(\lambda_{1}, \lambda_{2}\right)
$$

(see [10]). By the Spectral Mapping Theorem (see [3], p. 208) we get

$$
e^{\sigma(B)}=\sigma\left(e^{B}\right)=\left\{\lambda_{1}, \lambda_{2}\right\}
$$

Hence card $\sigma(B)=2$. Note that $\sigma(B)$ consists of real elements. In fact, suppose that $a+b \mathrm{i} \in \sigma(B)$ for some $a, b \in \mathbb{R}, b \neq 0$, then $\sigma(B)=\{a+b \mathrm{i}, a-b \mathrm{i}\}$. By (3.6) we have $e^{a+b \mathrm{i}} \in \mathbb{R}$. Thus $b=k \pi$ for some integer $k$ and $e^{a+b \mathrm{i}}=e^{a-b \mathrm{i}}$, so $e^{\sigma(B)}$ consists of one element $\left\{e^{a}\right\}$ or $\left\{-e^{a}\right\}$, but this is a contradiction. Therefore, $\sigma(B)=\left\{\mu_{1}, \mu_{2}\right\}$, where $\mu_{1}, \mu_{2} \in \mathbb{R}$ and by (3.6), $e^{\mu_{j}}=\lambda_{j}$ and $\lambda_{j}>0 j=1,2$. Hence there is a non-singular matrix $Q \in M_{2}(\mathbb{R})$ such that $Q^{-1} B Q=J_{0}\left(\mu_{1}, \mu_{2}\right)$. By (3.5) we get

$$
J_{0}\left(\lambda_{1}, \lambda_{2}\right)=J_{0}\left(e^{\mu_{1}}, e^{\mu_{2}}\right)=e^{J_{0}\left(\mu_{1}, \mu_{2}\right)}=e^{Q^{-1} B Q}=Q^{-1} J_{0}\left(\lambda_{1}, \lambda_{2}\right) Q .
$$

Hence $J_{0}\left(\lambda_{1}, \lambda_{2}\right) Q=Q J_{0}\left(\lambda_{1}, \lambda_{2}\right)$. It is easy to show that then $Q=\mu I$ for $\mu \in \mathbb{R}$ and in consequence $J_{0}\left(\mu_{1}, \mu_{2}\right)=Q^{-1} B Q=B$. Since $B=P^{-1} A P$ we get $A=P J_{0}\left(\mu_{1}, \mu_{2}\right) P^{-1}$. It is easy to verify that matrix $A$ defined by formula (3.1) satisfies (1.1).

Case (ii). Let $S$ be a matrix satisfying the assumptions of (ii) and let $P=[\mathbf{a}, \mathbf{b}], S \mathbf{a}=\lambda \mathbf{a}$ and $S \mathbf{b}=\lambda \mathbf{a}+\mathbf{b}$. Then $P^{-1} S P=J_{1}(\lambda, \lambda)$.

Suppose $A \in M_{2}(\mathbb{R})$ is such that $e^{A}=S$. Putting $B:=P^{-1} A P$, we get $e^{B}=J_{1}(\lambda, \lambda)$ and $e^{\sigma(B)}=\sigma\left(e^{B}\right)=\{\lambda\}$. Hence $\sigma(B)=\{\ln |\lambda|+\mathrm{i} k \pi, \ln |\lambda|-$ $\mathrm{i} k \pi\}$ for some $k \in \mathbb{Z}$. If $k \neq 0$ then there exists a matrix $D$ such that $D^{-1} B D=$ $J(\ln |\lambda|, k \pi)$. Since

$$
e^{J(a, b)}=e^{a} J(\cos b, \sin b), \quad a, b \in \mathbb{R}
$$

(see [10], p.13) we have $P^{-1} S P=J_{1}(\lambda, \lambda)=e^{B}=D e^{J(\ln |\lambda|, k \pi)} D^{-1}=$ $D|\lambda| I D^{-1}=|\lambda| I$, which contradicts the fact that $S \neq \lambda I$. Therefore, $k=0$ so $\{\lambda\}=e^{\sigma(B)}=\left\{e^{\ln |\lambda|}\right\}=\{|\lambda|\}$, which yields $\lambda>0$ and $\sigma(B)=\{\ln \lambda\}$. Since $B \neq(\ln \lambda) I$ there exists $Q$ such that

$$
Q^{-1} B Q=J_{1}(\ln \lambda, \ln \lambda) .
$$

Hence

$$
e^{J_{1}(\ln \lambda, \ln \lambda)}=e^{Q^{-1} B Q}=Q^{-1} e^{B} Q=Q^{-1} J_{1}(\lambda, \lambda) Q
$$


Since

$$
e^{J_{1}(a, a)}=e^{a} J_{1}(1,1) \quad \text { for } a \in \mathbb{R}
$$

(see [10]) we have $e^{J_{1}(\ln \lambda, \ln \lambda)}=\lambda J_{1}(1,1)=J_{\lambda}(\lambda, \lambda)$. Combining this and (3.9) we get $Q J_{\lambda}(\lambda, \lambda)=J_{1}(\lambda, \lambda) Q$. A simple calculation shows that $Q=J_{b}(\lambda a, a)$ for some $a, b \in \mathbb{R}, a \neq 0$. Thus by (3.8),

$$
\begin{aligned}
B & =Q J_{1}(\ln \lambda, \ln \lambda) Q^{-1}=J_{b}(\lambda a, a) J_{1}(\ln \lambda, \ln \lambda) J_{-\frac{b}{\lambda a^{2}}}(1 / \lambda a, 1 / a) \\
& =J_{\frac{1}{\lambda}}(\ln \lambda, \ln \lambda) .
\end{aligned}
$$

Thus $A=P J_{\frac{1}{\lambda}}(\ln \lambda, \ln \lambda) P^{-1}$. Conversely, applying (3.10) it is easy to verify that $e^{A}=S$.

Case (iii). Suppose $S=\lambda I$, where $\lambda \in \mathbb{R} \backslash\{0\}$ and let $A \in M_{2}(\mathbb{R})$ be such that $e^{A}=\lambda I$. Hence, by (3.6), $\sigma(A)=\{\ln |\lambda|+k \pi \mathrm{i}, \ln |\lambda|-k \pi \mathrm{i}\}$ for an odd $k$ if $\lambda>0$ and an even $k$ if $\lambda<0$.

Let $C$ be a Jordan form of $A$ that is $C=J(\ln |\lambda|, k \pi)$ for $k \in \mathbb{Z}$ or $C=J_{1}(\ln \lambda, \ln \lambda)$ and $P^{-1} A P=C$ for $P \in M_{2}(\mathbb{R})$. Notice that only the first case can occur. Indeed, if $C=J_{1}(\ln \lambda, \ln \lambda)$ then, by $(3.10)$, we have $\lambda I=e^{A}=e^{P J_{1}(\ln \lambda, \ln \lambda) P^{-1}}=P e^{J_{1}(\ln \lambda, \ln \lambda)} P^{-1}=\lambda P J_{1}(1,1) P^{-1}$ and in consequence $J_{1}(1,1)=I$, a contradiction. Therefore (3.3) holds with $k \in \mathbb{Z}$ and $P \in M_{2}(\mathbb{R})$.

Conversely, by (3.7) and (3.3), for every non-singular $P \in M_{2}(\mathbb{R})$ we have $e^{A}=\lambda I$ for odd $k$ if $\lambda<0$ and even $k$ for $\lambda>0$.

Case (iv). Let $\mathbf{a}=\operatorname{re} \mathbf{w}, \mathbf{b}=\operatorname{im} \mathbf{w}$, where $S \mathbf{w}=\lambda \mathbf{w}$ and $P:=[\mathbf{a}, \mathbf{b}]$. Then $P^{-1} S P=J(\alpha, \beta)$ where $\alpha=\operatorname{re} \lambda$ and $\beta=\operatorname{im} \lambda \neq 0$. Let $A \in M_{2}(\mathbb{R})$ and $e^{A}=S$. Put $B:=P^{-1} A P$, then

$$
e^{B}=P^{-1} e^{A} P=J(\alpha, \beta)
$$

and, by $(3.6), e^{\sigma(B)}=\sigma\left(e^{B}\right)=\sigma(J(\alpha, \beta))=\{\alpha+\mathrm{i} \beta, \alpha-\mathrm{i} \beta\}=\{\lambda, \bar{\lambda}\}$. Hence $\sigma(B)=\{\mu+\mathrm{i} \nu, \mu-\mathrm{i} \nu\}$, where

$$
\mu=\ln |\lambda|, \quad \nu=\operatorname{Arg} \lambda+2 k \pi
$$

for $k \in \mathbb{Z}$ and $\alpha=e^{\mu} \cos \nu, \beta=e^{\mu} \sin \nu$. Furthermore, similarly as for the matrix $S$, there exists a non-singular matrix $Q$ such that

$$
Q^{-1} B Q=J(\mu, \nu) \text {. }
$$

Thus, by (3.7), we have $J(\alpha, \beta)=e^{\mu} J(\cos \nu, \sin \nu)=e^{J(\mu, \nu)}=e^{Q^{-1} B Q}=$ $Q^{-1} J(\alpha, \beta) Q$, so $Q J(\alpha, \beta)=J(\alpha, \beta) Q$. The last relation implies that $Q=$ $J(x, y)$ for some $x, y \in \mathbb{R}$ and $J(x, y) J(\mu, \nu)=J(\mu, \nu) J(x, y)$. Therefore (3.13) becomes $B=J(\mu, \nu)$. Hence the fact that $A=P^{-1} J(\mu, \nu) P$ and (3.12) give (3.4).

Obviously, if $A$ is given by (3.4) then, by (3.7), we get $e^{A}=S$.

Remark 3.2. The part (i) is also true for real $n \times n$ matrices, where $n>2$. The proof is the same, it differs only in minor details. 
Open Access. This article is distributed under the terms of the Creative Commons Attribution License which permits any use, distribution, and reproduction in any medium, provided the original author(s) and the source are credited.

\section{References}

[1] Abate, M.: Discrete holomorphic local dynamical systems Holomorphic dynamical systems. Lect. Note. Math. 1998, 1-55 (2010)

[2] Balogh, A.: On the determination of geomertic objects with special transformation formulae. Math. Cluj 1(24), 199-120 (1959)

[3] Conway, J.B.: A course in functional analysis. Graduate Texts in Mathematics 96. Springer, New York (1985)

[4] Culver, W.J.: On the existence and uniqeness of the real logarithm of a matrix. Proc. Amer. Math. 17, 1146-1151 (1966)

[5] Hille, E., Philips, R.S.: Functional analysis and semi-groups. AMS Colloquium Publications, Vol. 31. Providence, Rhode Island (1957)

[6] Kuczma, M., Kucharzewski, M.: General solutions of the functional equation $f(x y)=$ $f(x) f(y)$ for second degree matrices $f$, [Polish] Zeszyty Nauk. Wyz. Szkoły Ped. w Katowicach Mat 3, 61-70 (1962)

[7] Kuczma, M.: Functional equations in a single variable. Monografie Mat. 46, PWN, Warszawa (1968)

[8] Kuczma, M.: Note on linearization. Ann.Polon. Math. 29, 75-81 (1974)

[9] Kuczma, M., Choczewski, B., Ger, R.: Iterative functional equations.Encyclopaedia of Mathematics and its Applications, 32. Cambridge University Press, Cambridge (1990)

[10] Perko, L.: Differential equations and dynamical systems. Texts in Applied Mathematics, 7. Springer, New York (2001)

[11] Robinson, C.: Dynamical systems. Stability, symbolic dynamics and chaos. Studies in Advanced Mathematics. CRC Press, Boca Raton, FL (1995)

[12] Sherif, N., Morsy, E.: Computing real logarithm of real matrix. Int. J. Alg. 2(3), 131$142(2008)$

[13] Smajdor, W.: Formal solutions of a functional equation. Zeszyty Nauk. Uniw. Jagiello. Prace Mat. Zeszyt 13, 71-78 (1969)

[14] Sternberg, S.: Local contractions and a theorem of Poincaré. Am. J. Math. 79, 809824 (1957)

[15] Walter W.: Ordinary differential equations. Graduate Texts in Mathematics, 182. Readings in Mathematics. Springer, New York (1998)

[16] Zdun, M.C.: On a limit formula for embeddings of diffeomorphisms in regular iteration semigroups. J. Differ. Equ. Appl. 19(6), 942-951 (2013)

[17] Zdun, M.C.: On the Schröder equation and iterative sequences of $C^{r}$ diffeomophisms in $\mathbb{R}^{n}$ spaces. Aequ. Math. 85, 1-5 (2013)

Marek Cezary Zdun and Paweł Solarz

Institute of Mathematics

Pedagogical University

ul. Podchorążych 2

30-084 Kraków

Poland

e-mail: mczdun@up.krakow.pl 


\section{Paweł Solarz}

e-mail: psolarz@up.krakow.pl

Received: March 31, 2014

Revised: May 9, 2014 